\title{
Four-Component Analysis and Modeling of Positron Annihilation Lifetime Parameters in AOT/Water/Isooctane Microemulsions
}

\author{
M. F. Ferreira Marques \\ Instituto Superior de Engenharia, 3000 Coimbra, Portugal
}

\author{
H. D. Burrows, ${ }^{\dagger}$ M. da Graça Miguel, ${ }^{\dagger}$ A. P. de Lima, ${ }^{\ddagger}$ and C. Lopes Gil ${ }^{\ddagger}$ \\ Departamentos de Química e de Física da Universidade, 3000 Coimbra, Portugal
}

\author{
G. Duplâtre* \\ Centre de Recherches Nucléaires, IN2P3/CNRS, Chimie Nucléaire, B.P. 28, 67037, Strasbourg Cedex, France
}

Received: September 25, 1995; In Final Form: January 8, $1996^{\otimes}$

\begin{abstract}
Reverse micelles are studied in sodium AOT/water/isooctane mixtures as functions of AOT concentration $\left(C_{\mathrm{AOT}}\right)$, water to AOT mole ratio $\left(w_{0}\right)$ and temperature $(T)$, from 294 to $333 \mathrm{~K}$, using positron annihilation lifetime spectroscopy (LS). By four-component analysis of the spectra, it is possible to extract the LS parameters (intensities, $I_{i}$, and lifetimes, $\tau_{i}$ ) of the triplet positronium (o-Ps) present in the aqueous $\left(I_{3}, \tau_{3}\right)$ and organic $\left(I_{4}, \tau_{4}\right)$ phases. The latter lifetime is constant and corresponds with the value measured in pure isooctane, while $\tau_{3}$ is remarkably lower than the value for pure water. This difference is attributed to the out-diffusion of o-Ps from the water cores to isooctane. The relevant rigorous diffusion equations imply two fitting parameters, the radius of the water aggregates $\left(r_{0}\right)$ and a transmission factor $(h)$. Fixation of $r_{0}=3.6$ $\mathrm{nm}$ for $C_{\mathrm{AOT}}=0.1 \mathrm{M}, w_{0}=20$, and $T=294 \mathrm{~K}$, as known from previous work, allows the quantitative derivation of the $r_{0}$ values for all other conditions. The water spheres appear to present some permeability to o-Ps, with a transmission factor $h=0.12 \mathrm{~nm}^{-1}$. The sphere radius increases smoothly with $C_{\mathrm{AOT}}$ and $w_{0}$ and, more importantly, with $T$. The changes with $w_{0}$ give $r_{0}=0.181 w_{0}$ and $0.186 w_{0} \mathrm{~nm}$ at 294 and $298 \mathrm{~K}$, respectively, and are in excellent agreement with previous proposals. The sum of the intensities, $I_{\text {tot }}=I_{3}+$ $I_{4}$, is much lower than the o-Ps intensity in pure isooctane. In particular, at $C_{\mathrm{AOT}}>0.04 \mathrm{M}, I_{\text {tot }}$ appears very close to the value found for pure water. The possibility of a strong inhibition of Ps formation due to the micelles, as proposed in previous work, is ruled out because of the negligible electron or positron scavenging ability of alkyl sulfonates. It is thus concluded that Ps formation occurs primarily in the aqueous part of the micelles, the water aggregates representing efficient traps for the positrons while they are slowing down in the solutions.
\end{abstract}

\section{Introduction}

Positronium (Ps), the bound state of a positron $\left(\mathrm{e}^{+}\right)$with an electron $\left(\mathrm{e}^{-}\right)$, has been used increasingly in recent decades as an efficient probe of the physical and chemical properties of matter. ${ }^{1}$ The most commonly used positron annihilation technique (PAT) is lifetime spectroscopy (LS), which allows us to obtain both the lifetimes $\left(\tau_{i}\right)$ and relative abundances (or intensities, $I_{i}$ ) of the various positron species. Normally, in order of increasing lifetimes, these are singlet Ps ( $\mathrm{p}-\mathrm{Ps}, i=1)$, free positron $(i=2)$, and triplet Ps (o-Ps, $i=3$ ). Because of its distinct long lifetime, the latter appears to be the most useful probe. The o-Ps decay occurs with the emission of two $\gamma$-rays through the pick-off process, i.e., the annihilation of the positron with one of the (bound) electrons of the surrounding medium. Information is obtained through two distinct, independent processes, Ps formation and annihilation, both of which depend on various characteristics of the systems under investigation. In liquids, Ps formation may occur on a very short time scale (ca. picoseconds) and involves the reaction of $\mathrm{e}^{+}$with one of the electrons released by ionization at the end of the positron track in a spur containing a variety of labile, reactive species

\footnotetext{
Departamento de Química da Universidade.

* Departamento de Física da Universidade.

${ }^{\otimes}$ Abstract published in Advance ACS Abstracts, April 1, 1996.
}

such as quasi-free electrons and holes. ${ }^{2,3}$ The nature of the solvent, the presence of solutes (e.g., electron or hole scavengers), and various physicochemical parameters, such as temperature, can affect Ps formation. Once formed, Ps can be influenced in various ways by the medium, particularly through reactions with solutes, ${ }^{4}$ eventually leading to a decrease in its lifetime.

Although Ps has proven to be very useful in a large variety of areas, ${ }^{1}$ particular attention has been given to the study of complex, multicomponent media ${ }^{1}$ and, more specifically, to micellar systems. ${ }^{5-8}$ By their nature, a detailed understanding of the structure and properties of such systems demands combined study by several techniques, among which LS appears promising. However, it must be acknowledged that, notwithstanding the intrinsic interest of the work produced so far, particularly as concerns the behavior of the probe itself, little precise quantitative information on micelles has been extracted from experimental PAT data. A major reason for this lies in the limited time resolution and counting efficiency of the LS apparatus until only recently. A medium, such as a micellar solution, containing several pseudophases is expected to result in the existence of a corresponding set of Ps states, whose respective lifetimes and intensities are difficult to extract from LS spectra without sufficient resolution and integration of counts. However, taking into account recent developments in 
LS electronics and detectors, it appears that more precise information, although still difficult to obtain, could be derived from LS experiments, both on the behavior of Ps in micellar systems and on their structure and properties.

Therefore, by taking advantage of these improvements in LS devices, the present work aims to deepen the knowledge and understanding of both the probe, Ps, and a complex medium. In a first step, the latter consists of inverse micelles formed in aqueous solutions of AOT in an organic nonpolar solvent, isooctane. This system has been previously studied to some extent through various techniques, ${ }^{6,9-12}$ so that a comparison could be established with the results to come from LS. However, relatively few studies have been presented on the temperature effects in these systems. The parameters investigated here are AOT concentration, water/AOT ratio $\left(w_{0}\right)$, and temperature $(T)$.

\section{Experimental Section}

AOT (sodium di-2-ethylhexylsulfosuccinate, Sigma) was dissolved in methanol and filtered with alumina, and the solvent was evaporated. ${ }^{13}$ The AOT/water/isooctane system was studied at constant $\mathrm{AOT}$ concentration $\left(C_{\mathrm{AOT}}=0.1 \mathrm{M}\right)$, with varying $w_{0}$ values $\left(w_{0}=\left[\mathrm{H}_{2} \mathrm{O}\right] /[\mathrm{AOT}]\right)$ from 10 to 40 , at different temperatures. In another series of experiments, $C_{\mathrm{AOT}}$ was varied from 0.02 to $0.1 \mathrm{M}$, with constant $w_{0}=20$ at $294 \mathrm{~K}$. The $w_{0}$ values were determined by assuming ca. 1 water molecule of hydration in the pure AOT. ${ }^{14}$

The LS experiments were carried out in parallel in the Coimbra and Strasbourg laboratories. In both cases, the positron source consisted of ca. $7 \times 10^{5} \mathrm{~Bq}$ of ${ }^{22} \mathrm{Na}$ diffused in a thin sodium glass film sustained by an all-glass holder, yielding a source correction of about $10 \% .^{15}$ The source was immersed in the solution to be studied, which had been placed in a Pyrex tube previously degassed by the usual freeze-thaw technique to avoid any interference due to oxygen. The LS devices consisted of $\mathrm{BaF}_{2}$ or mixed plastic $\mathrm{NE} 111 / \mathrm{BaF}_{2}$ scintillators, with time resolutions of 170 and $250 \mathrm{ps,} \mathrm{in} \mathrm{Coimbra} \mathrm{or}$ Strasbourg, respectively. In preliminary experiments (e.g., pure isooctane and water) the integrated counts did not exceed ca. 5 $\times 10^{5}$. For the AOT/water/isooctane measurements longer counting periods were necessary. However, to overcome the non-Poisson distribution of the counts in the channels of the LS spectra, the integrated counts were kept within the range 5 $\times 10^{5}$ to $1.4 \times 10^{6}$.

The temperature range $(294-333 \mathrm{~K})$ was investigated by using thermostatically controlled devices $( \pm 0.5 \mathrm{~K})$. The experiments in Coimbra were carried out at $298 \mathrm{~K}$, with each sample being counted several times (typically, six) for a better definition of the various LS parameters. Similar repeated countings were made in Strasbourg at $294 \mathrm{~K}$, while at higher temperatures the samples were only counted 1-3 times, resulting in a lower quality of the derived LS parameters.

For the pure solvents, the LS spectra were analyzed in terms of three components. On physical grounds in the AOT/water/ isooctane mixtures, $\mathrm{p}-\mathrm{Ps}$, free $\mathrm{e}^{+}$, and o-Ps should each be present in two states, corresponding to the two phases present in the solution. However, due to the limited resolution, it is unrealistic to expect to be able to distinguish between the components corresponding to the two pairs of short-lived species, p-Ps and $\mathrm{e}^{+}$, in isooctane and in the micelles. Therefore, only three- and four-component analyses were attempted, with the latter hopefully allowing us to discriminate between the two longest lived o-Ps states. In the following, subscripts $1-3$ (and 4) of the LS parameters, $I_{i}$ and $\tau_{i}$, will be used in the order of increasing lifetimes, thus corresponding to
p-Ps, free $\mathrm{e}^{+}$, and o-Ps (in two states, when present), respectively. Values for the pure solvents, water and isooctane, will be denoted by superscripts aq and is. The decay rate constants, $1 / \tau_{i}$, will be denoted as $\lambda_{i}$.

For the pure solvents, water and isooctane, the experimental error was within $20 \mathrm{ps}$ and $0.2 \%$ for the o-Ps lifetime and intensity, respectively. In the four-component analyses of the spectra for the AOT/water/isooctane mixtures with all parameters free, the errors for the longest lived o-Ps state parameters, $\tau_{4}$ and $I_{4}$, were only slightly higher, at about $30 \mathrm{ps}$ and $0.3 \%$, respectively. However, for the second longest lived component, $\tau_{3}$, attributed to o-Ps in the aqueous phase of the inverted micelles, the experimental errors were higher, due to the somewhat low intensity of this component in all cases: the error in $\tau_{3}$ ranged from about 30 up to $100 \mathrm{ps}$ and that in $I_{3}$ from 0.5 up to $1 \%$, depending on the number of repeated countings for each sample. The differences in $\chi^{2}$ between three- and fourcomponent analyses usually were not significant, except for those micellar systems having the highest water contents. The four-component analysis was chosen anyway due to its physical validity.

\section{Results and Discussion}

A. Pure Solvents. Pure isooctane was investigated as a function of the temperature up to $333 \mathrm{~K}$ : $\tau_{1}^{\text {is }}$ was constant at $122 \pm 7$ ps. This is close to the value of 125 ps known for the intrinsic p-Ps decay in a vacuum, but somewhat lower than the 137 ps expected from the contact density parameter measured when applying an external magnetic field in LS experiments. ${ }^{16}$ As shown in Figure 1, all other LS parameters show an increase with $T$, the values at $294 \mathrm{~K}$ agreeing well with those of previous determinations. ${ }^{16,17}$ Fitting of the data on the basis of linear dependence on $T$ (Kelvin) leads to the following equations, with standard deviations $(\sigma)$ well within the experimental errors:

$$
\begin{gathered}
\tau_{2}^{\text {is }}(\mathrm{ps})=0.505 T+288.6(\sigma=1.67 \mathrm{ps}) \\
\tau_{3}^{\text {is }}(\mathrm{ps})=10 T+1151(\sigma=16 \mathrm{ps}) \\
I_{3}^{\text {is }}(\%)=\left(8.55 \times 10^{-2}\right) T+21.66(\sigma=0.11 \%)
\end{gathered}
$$

From values previously published for pure water, ${ }^{18,19}$ the following linear expressions can be derived:

$$
\begin{gathered}
\tau_{3}^{\mathrm{aq}}(\mathrm{ps})=-1.569 T+2271 \\
I_{3}^{\mathrm{aq}}(\%)=\left(2.769 \times 10^{-2}\right) T+19.72
\end{gathered}
$$

Our value at $294 \mathrm{~K}$ was lower than that expected from eq 5, with $I_{3}^{\mathrm{aq}}=27.1 \%$.

B. Lifetimes in AOT/Water/Isooctane Mixtures. In threecomponent analysis of the LS spectra, the longest lifetime due to o-Ps decay in either subphase at each temperature appears to be slightly lower than $\tau_{3}^{\text {is }}$, irrespective of the other variables, $C_{\mathrm{AOT}}$ and $w_{0}$. The mean o-Ps lifetime should be lower than $\tau_{3}^{\text {is }}$, as some Ps atoms should decay in the aqueous core of the micelles where $\tau_{3}^{\text {aq }} \ll \tau_{3}^{\text {is }}$. However, due to the rather small volume fraction of the aqueous cores (a few percent at most), ${ }^{20}$ the mean o-Ps lifetime is expected to be only slightly lower than $\tau_{3}^{\text {is }}$, as is verified experimentally. Furthermore, the small change observed is in agreement with the expectation of little chemical reactivity of Ps toward either the organic tails or the polar sulfonate heads of the AOT molecules. Preliminary experiments on sodium methanesulfonate in water show that neither $\tau_{3}^{\mathrm{aq}}$ nor $I_{3}^{\mathrm{aq}}$ is significantly affected by this compound, 


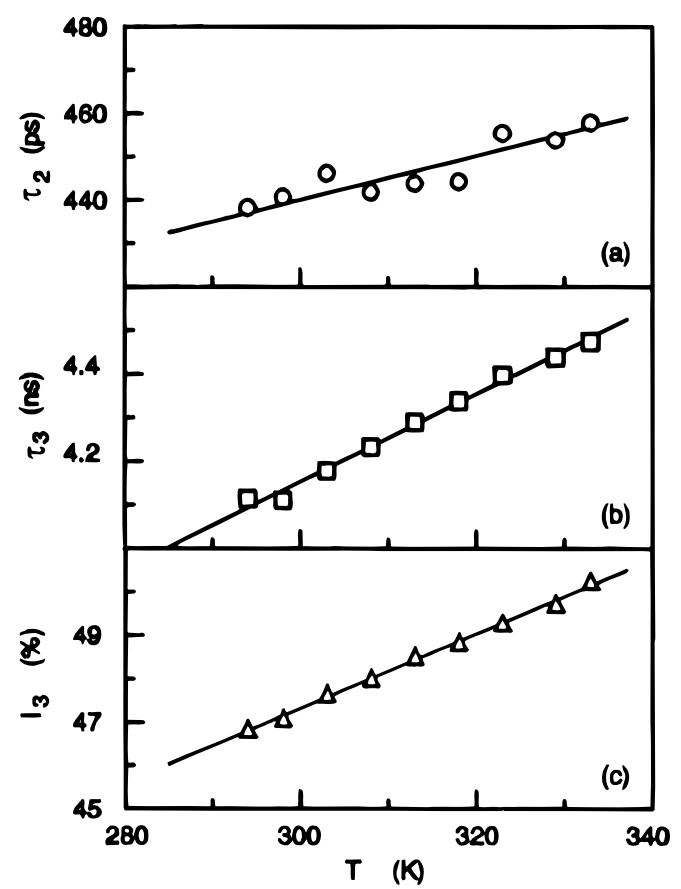

Figure 1. Variation in the LS parameters for pure isooctane as a function of temperature, $T$ (Kelvin): (a) $\tau_{2}$ (ps); (b) $\tau_{3}$ (ns); (c) $I_{3}(\%)$.

which, as it does not form micelles, represents a reliable surrogate for the AOT molecules. As a rule, only those solutes presenting high chemical reactivity are able to interact efficiently, at moderate concentrations, with Ps within its lifetime. ${ }^{3}$

In a second step, therefore, in an attempt to gain more detailed information on both the probe and the solutions, the LS spectra were analyzed in terms of four components. In all free parameter analyses, for all $C_{\mathrm{AOT}}, w_{0}$, and $T$, the longest lifetime, $\tau_{4}$, appears to be very close (within $1-2 \sigma$ ) to $\tau_{3}^{\text {is }}$, confirming that o-Ps in isooctane is not affected by the presence of AOT.

Curiously enough, whether all parameters are set as free or $\tau_{4}$ is fixed to the value of $\tau_{3}^{\text {is }}, \tau_{2}$ in the mixtures systematically appears lower than either $\tau_{2}^{\text {is }}$, as expressed by eq 1 , or $\tau_{2}^{\text {aq }}$, which increases smoothly from about 400 to $420 \mathrm{ps}$ in the temperature range studied. ${ }^{18,19}$ No significant influence of either $C_{\mathrm{AOT}}$ or $w_{0}$ is observed, and only some smooth changes with $T$ (Kelvin) can be defined:

$$
\tau_{2}(\mathrm{ps})=0.480 T+222.6(\sigma=4.5 \mathrm{ps})
$$

The reasons for the lower values of $\tau_{2}$ than $\tau_{2}^{\mathrm{aq}}$ and $\tau_{2}^{\mathrm{is}}$ are not yet clear, although some lowering due to interference with the shortest lifetime, $\tau_{1}$, cannot be excluded. In contrast with the similarity between $\tau_{4}$ and $\tau_{3}^{\text {is }}, \tau_{3}$ appears to be significantly lower than $\tau_{3}^{\text {aq }}$, although always remaining well above $\tau_{2}^{\text {aq }}$ or $\tau_{2}^{\text {is }}$. For a given sample, the spread of experimental values of $\tau_{3}$ is rather large when all LS parameters are allowed to be free, but is more coherent when $\tau_{4}$ is fixed to the value found for $\tau_{3}^{\text {is }}$ at a specified $T: \tau_{3}$ varies roughly in the range 900-1300 ps and increases with $C_{\mathrm{AOT}}, w_{0}$ or $T$ (see Table 1). Note that for $C_{\mathrm{AOT}}=0.1 \mathrm{M}$ and $w_{0}=30$ at $333 \mathrm{~K}$, the LS parameters were exactly those of pure isooctane, denoting breakup of the micellar aggregates.

A decrease in its lifetime implies that Ps is affected by some physicochemical process, most commonly either a chemical reaction or trapping. In the present case, since the AOT molecules are not likely to promote any reaction with Ps, as verified with sodium methanesulfonate, a plausible explanation is the out-diffusion of the Ps atoms from the aqueous core of
TABLE 1: LS Parameters for the Various AOT/Water/ Isooctane Systems, as Functions of $C_{\mathrm{AOT}}, w_{0}$, and $T^{a}$

\begin{tabular}{lccccccccc}
\hline $\begin{array}{c}C_{\mathrm{AOT}} \\
(\mathrm{M})\end{array}$ & $\begin{array}{c}T \\
(\mathrm{~K})\end{array}$ & $w_{0}$ & $\begin{array}{c}I_{3} \\
(\%)\end{array}$ & $\begin{array}{c}I_{4} \\
(\%)\end{array}$ & $\begin{array}{c}I_{\mathrm{tot}} \\
(\%)\end{array}$ & $\begin{array}{c}\tau_{3} \\
(\mathrm{ps})\end{array}$ & $\begin{array}{c}D_{\mathrm{Ps}}, \\
\left(\mathrm{nm}^{2} \mathrm{~ns}^{-1}\right)\end{array}$ & $\begin{array}{c}r_{0} \\
(\mathrm{~nm})\end{array}$ & $\begin{array}{c}k \\
\left(\mathrm{M}^{-1}\right)\end{array}$ \\
\hline 0.02 & 294 & 20 & 13.0 & 20.3 & 33.3 & 830 & 4.15 & 2.19 & \\
0.04 & & & 13.1 & 17.8 & 30.9 & 880 & & 2.43 & \\
0.06 & & & 12.0 & 16.2 & 28.2 & 912 & & 2.65 & \\
0.08 & & & 5.7 & 19.4 & 25.1 & 1048 & & 3.46 & \\
0.1 & & & 6.9 & 19.3 & 26.2 & 1067 & & $3.60^{b}$ & \\
0.1 & 294 & 10 & 6.5 & 21.0 & 27.5 & 838 & 4.15 & 2.23 & 0.03 \\
& & 20 & 6.9 & 19.3 & 26.2 & 1067 & & $3.60^{b}$ & 0.13 \\
& & 30 & 5.6 & 18.4 & 24.0 & 1242 & & 5.30 & 0.47 \\
& 298 & 10 & 4.4 & 21.4 & 25.8 & 1007 & 4.59 & 3.49 & 0.13 \\
& 15 & 5.5 & 20.5 & 26.0 & 1048 & & 3.81 & 0.15 \\
& 20 & 7.0 & 19.6 & 26.6 & 1057 & & 3.88 & 0.12 \\
& 30 & 5.2 & 18.7 & 24.9 & 1239 & & 5.80 & 0.53 \\
& & 40 & 5.7 & 17.7 & 23.4 & 1278 & & 6.36 & 0.69 \\
& 303 & 10 & 4.8 & 21.1 & 25.9 & 1046 & 5.25 & 4.32 & 0.13 \\
& 20 & 7.6 & 19.8 & 27.4 & 961 & & 3.62 & 0.06 \\
& 30 & 6.9 & 19.2 & 26.1 & 1206 & & 6.12 & 0.29 \\
& 313 & 10 & 5.3 & 21.6 & 26.9 & 1084 & 6.62 & 5.85 & 0.12 \\
& 20 & 4.8 & 19.7 & 24.5 & 1257 & & 8.62 & 0.44 \\
& 30 & 6.8 & 20.3 & 27.1 & 1103 & & 6.09 & 0.24 \\
& 323 & 10 & 5.3 & 21.7 & 27.0 & 1079 & 8.16 & 7.06 & 0.13 \\
& 20 & 3.7 & 21.3 & 25.0 & 1191 & & 9.02 & 0.58 \\
& 30 & 5.2 & 22.0 & 27.2 & 1185 & & 8.90 & 0.48 \\
& 333 & 10 & 4.1 & 22.0 & 26.1 & 1171 & 9.86 & 10.3 & 0.25 \\
& 20 & 5.7 & 21.7 & 27.4 & 1120 & & 9.20 & 0.26 \\
& 30 & demixed & & & & & &
\end{tabular}

${ }^{a} D_{\mathrm{Ps}}$ is the Ps diffusion coefficient calculated from eq $8, r_{0}$ is the water core radius derived from the values of $\tau_{3}$ by using eq A9 with $h$ $=0.1205 \mathrm{~nm}-1$, and $k$ is the inhibition constant from eq A12. Representative values of intensities and lifetimes for pure solvents calculated from eqs $2-5$ at $294 \mathrm{~K}$ are $\tau_{3}^{\text {is }}=4092 \mathrm{ps}, I_{3}^{\text {is }}=46.8 \%$, $\tau_{3}^{\mathrm{aq}}=1809 \mathrm{ps}$, and $I_{3}^{\mathrm{aq}}=27.9 \% .{ }^{b}$ Fixed value.

the micelles to the surrounding solvent, isooctane. By using superscripts aq and is to distinguish the two states of Ps, the overall process can be described as follows:

$$
2 \gamma \stackrel{\lambda_{3}^{\mathrm{aq}}}{\longrightarrow} \mathrm{Ps}^{\mathrm{aq}} \stackrel{k_{\text {out }}}{\longrightarrow} \mathrm{Ps}^{\text {is }} \stackrel{\lambda_{3}^{\text {is }}}{\longrightarrow} 2 \gamma
$$

where $\lambda_{3}^{\mathrm{aq}}=1 / \tau_{3}^{\mathrm{aq}}$ and $\lambda_{3}^{\mathrm{is}}=1 / \tau_{3}^{\mathrm{is}}$ are the pick-off decay rate constants in water and isooctane, respectively, and $k_{\text {out }}$ expresses the outdiffusion process.

By approximating the aqueous cores to spheres of radius $r_{0}$, it is possible to obtain a general equation for $\mathrm{Ps}^{\mathrm{aq}}$ as a function of time $(t) .^{21}$ In a next step, the equation representative of the LS spectra, given by $\mathrm{d}(2 \gamma) / \mathrm{d} t$, can be derived, and both the second longest lived lifetime, $\tau_{3}$, and the intensities, $I_{3}$ and $I_{4}$, for the AOT/water/isooctane mixtures can be expressed quantitatively. For the sake of clarity, these equations are given in the Appendix.

The rigorous mathematical expression for $\tau_{3}$ derived on the basis of eq 7 includes $r_{0}$, the Ps ${ }^{\text {aq }}$ diffusion constant, $D_{\mathrm{Ps}}$, and a transmission factor, $h$, which is negligible for an impermeable sphere and tends to infinity for a permeable sphere.

The Ps diffusion constant in water can be evaluated to a good approximation by the Stokes-Einstein expression as

$$
D_{\mathrm{Ps}}=k_{\mathrm{B}} T /\left(6 \pi r_{\mathrm{Ps}} \eta\right)
$$

where $k_{\mathrm{B}}$ is the Boltzmann constant, $T$ is the absolute temperature, $r_{\mathrm{Ps}}=0.053 \mathrm{~nm}$ is the Ps radius, and $\eta$ is the viscosity. Table 1 gives the calculated values of $D_{\mathrm{Ps}}$ at each temperature. It may be noted that when the Ps reaction rate constant with solutes is calculated on the basis of eq 8 , very good agreement is found with the highest experimental values, representative of purely diffusion-controlled reactions (e.g., refs 3 and 22), 


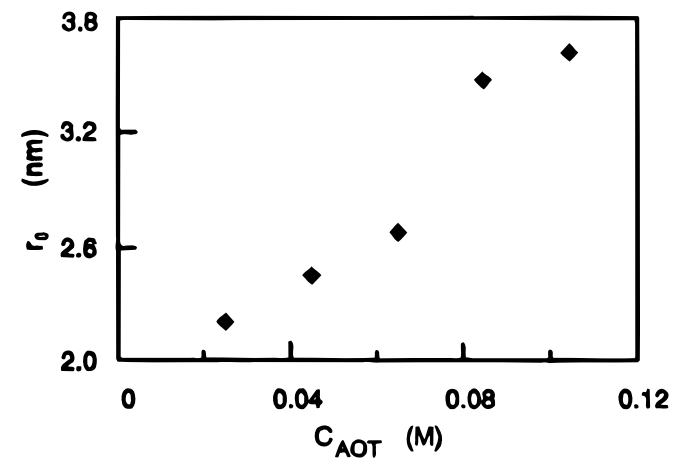

Figure 2. Variation in the water core radius of the micelles, $r_{0}(\mathrm{~nm})$, determined from LS measurements, as a function of AOT concentration in isooctane, $C_{\mathrm{AOT}}(\mathrm{M})$.

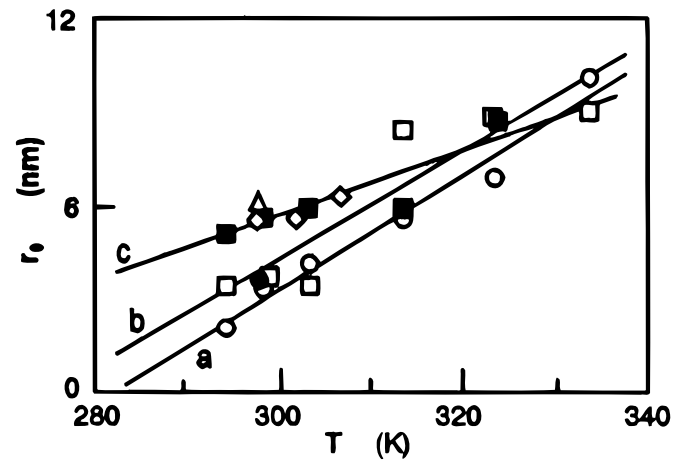

Figure 3. Variation in the water core radius of the micelles, $r_{0}(\mathrm{~nm})$, as a function of temperature, $T(\mathrm{~K})$, for various values of the water/ AOT mole ratio: $w_{0}=10(\bigcirc), 15(\bullet), 20(\square), 30(\square)$, and $40(\triangle)$. The solid lines are linear fittings for $w_{0}=10(\mathrm{a}), 20(\mathrm{~b})$, and $30(\mathrm{c})$ (see text). The values for $w_{0}=32.6(\diamond)$ are for $\mathrm{AOT} / \mathrm{D}_{2} \mathrm{O} /$ decane mixtures and taken from ref 27.

which gives confidence in the validity of the application of this equation to these processes.

To calculate $\tau_{3}$ at specified values of $C_{\mathrm{AOT}}, w_{0}$, and $T$, one is left with two fitting parameters, $r_{0}$ and $h$. In the absence of any established correlation for $r_{0}$ or $\mathrm{h}$ with either $\mathrm{C}_{\mathrm{AOT}}, w_{0}$, or $T$, it is unrealistic to fit each distinct experimental value of $\tau_{3}$ with two parameters. Therefore, the set of $\tau_{3}$ values was analyzed on the basis of the following two assumptions: (i) $h$ is independent of the various conditions $\left(C_{\mathrm{AOT}}, w_{0}, T\right)$, and (ii) the water core radius at $T=294 \mathrm{~K}, C_{\mathrm{AOT}}=0.1 \mathrm{M}$, and $w_{0}=$ 20 is $r_{0}=3.6 \mathrm{~nm}$, a value that appears reliable as has been found repeatedly by using various techniques. ${ }^{23-25}$ For the preceding conditions, with $\tau_{3}=1067 \mathrm{ps}$ (Table 1 ), a value of $h=0.1205 \mathrm{~nm}^{-1}$ was obtained and fixed thereafter in eq A8 to derive the other values of $r_{0}$, whose variations with $C_{\mathrm{AOT}}, w_{0}$, and $T$ are shown in Figures 2 and 3.

The micelle radius appears, as expected, to increase with $w_{0}$. For $C_{\mathrm{AOT}}=0.1 \mathrm{M}$ at $294 \mathrm{~K}$, the series $r_{0}=2.23,3.6$, and 5.30 $\mathrm{nm}$ for $w_{0}=10,20$, and 30 is in very good agreement with previous determinations, as, for example, $r_{0}=2.14,3.6$, and $5.0 \mathrm{~nm}$ in isooctane and 1.96, 3.30, and $4.67 \mathrm{~nm}$ in decane at $294 \mathrm{~K} .^{24}$ Similarly, the corresponding gross variations $r_{0}(\mathrm{~nm})$ $=0.181 w_{0}$ and $r_{0}(\mathrm{~nm})=0.186 w_{0}$ deduced from the data in Table 1 at 294 and $298 \mathrm{~K}$, respectively, agree well with previous empirical values of about $0.18-0.20 \mathrm{~nm}$ for the $r_{0} / w_{0}$ ratio. ${ }^{14,23,25}$ For fixed $w_{0}=20$ at $294 \mathrm{~K}$, at low AOT concentrations $r_{0}$ increases smoothly with $C_{\mathrm{AOT}}$ (Table 1 ), which is in qualitative agreement with a previous work at $w_{0}=5.4,{ }^{26}$ giving

$$
r_{0}(\mathrm{~nm})=19.25 C_{\mathrm{AOT}}(\mathrm{M})+1.711
$$

With the preceding observations, it appears that the assumption of the independence of $h$ from $C_{\mathrm{AOT}}$ and $w_{0}$ is quite plausible. The value fixed at $r_{0}=3.6 \mathrm{~nm}$ for $w_{0}=20$ and $C_{\mathrm{AOT}}=0.1 \mathrm{M}$ corresponds strictly to the aqueous core. If we suppose that $\mathrm{Ps}^{\mathrm{aq}}$ out-diffusion occurs outside of the water pool of the microemulsion droplets, with an additional size of about $0.5 \mathrm{~nm}$ (corresponding to the AOT headgroups), a radius of $r_{0}$ $=4.1 \mathrm{~nm}$ is expected. On this basis, a value of $h=0.141$ $\mathrm{nm}^{-1}$ is derived, leading to $r_{0}=2.55$ and $6.00 \mathrm{~nm}$ for $w_{0}=10$ and 30, respectively. Both values appear to be higher than those deduced for the aqueous core from other techniques, such as neutron scattering and density measurements. ${ }^{23-25}$

Another parameter that may be considered in eq A8 is $\lambda_{3}^{\text {aq }}$, which, in calculating the $r_{0}$ values given in Table 1, was fixed to the values predicted by eq 4 . As will be shown later, the intensities $I_{3}$ and $I_{4}$ strongly indicate that Ps formation occurs predominantly in aqueous medium. The specific properties of the water molecules in the micelles might be suspected to result in a modified pick-off lifetime for $\mathrm{Ps}^{\mathrm{aq}}$. However, this has to be discarded for the following reasons: (i) the o-Ps pick-off decay essentially depends on the average electron density at the positron site, which should not be substantially affected if water is enclosed in microemulsion droplets; and (ii) $\lambda_{3}^{\text {aq }}$ would be expected to increase, leading to similar effects, in relation to the calculation of $r_{0}$, as obtained by including headgroups (see $\Lambda_{\mathrm{n}}$ in eq A1).

There is little literature data on the behavior of $r_{0}$ as a function of $T$. Although low-frequency permittivity measurements show that droplet shapes may be temperature independent, the droplets may cluster. ${ }^{11}$ Further, dynamic light scattering studies suggest that droplet sizes may increase with $T .{ }^{9}$ From the data in Table 1 and in Figure 3 for $C_{\mathrm{AOT}}=0.1 \mathrm{M}, r_{0}$ increases significantly with $T$ in an approximately linear way in the $T$ range studied. Although the linear relationship for $w_{0}=20$ is less evident, this data was also fitted to a straight line to enable comparison with data at $w_{0}=10$ and $w_{0}=30$. The slope of the variations appears to be rather higher for $w_{0}=10$ than for $w_{0}=30$, so that at about $333 \mathrm{~K}$ the $r_{0}$ values for the various $w_{0}$ values appear to converge. One possible explanation for the increase in $r_{0}$ with $T$ is the coalescence of the micellar spheres upon encounter, which becomes more and more efficient as $T$ increases. The convergence may be explained qualitatively by the existence of an optimum size of the micelles due to the surface density of charges, which becomes independent of $w_{0}$ as $T$ increases.

Quantitatively, the variations in $r_{0}$ with $T$ (Kelvin), up to 333 $\mathrm{K}$, would be $r_{0}(\mathrm{~nm})=a_{T} T+b_{T}$, with $a_{T}=0.188,0.174$, and $0.107 \mathrm{~nm} \mathrm{~K}^{-1}$ and $b_{T}=-52.79,-47.63$, and $-26.24 \mathrm{~nm}$ for $w_{0}=10,20$, and 30, respectively. However, it must be noted that some dependence of the transmission factor, $h$, on $T$ may be expected inasmuch as the thermal energy should help passage across the potential barrier at the water-isooctane interface.

In contrast to the present results, the slope of the dependence of $r_{0}$ with $T$ found by neutron scattering for AOT/ $\mathrm{D}_{2} \mathrm{O} /$ decane $^{27}$ is suggested to increase with $w_{0}$. Curiously, however, the values $r_{0}=5.7,5.8$, and $6.5 \mathrm{~nm}$ for $w_{0}=32.64$ at 298, 301.6, and $306.5 \mathrm{~K}$ in this work fit very well in our data, as shown in Figure 3.

On energetic grounds, by considering the bubble states occupied by Ps in either solvent (e.g., ref 22), the out-diffusion of Ps from water to isooctane seems unfavorable. Doppler broadening ${ }^{16,18}$ or angular correlation ${ }^{17}$ experiments allow us to derive the full width at half-maximum, $\Gamma_{1}$, of the momentum distribution of the narrowest component, p-Ps. Since this species decays in an intrinsic mode, $\Gamma_{1}$ directly reflects the average kinetic energy of the Ps center of mass at the moment of annihilation, $E_{\mathrm{kin}}{ }^{28}$ 


$$
\Gamma_{1}=2\left(m_{0} c^{2} E_{\mathrm{kin}}\right)^{1 / 2}
$$

where $m_{0} c^{2}=511 \mathrm{keV}$. Typical available values for $\Gamma_{1}$ are $0.99^{18}-0.83 \mathrm{keV}^{17}$ in water and $0.64^{17}-0.60 \mathrm{keV}^{16}$ in isooctane, giving kinetic energies of about $0.4 \mathrm{eV}$ in water and $0.2 \mathrm{eV}$ in isooctane. On these grounds, the virial theorem would predict potential energies for Ps in the bubble state of about twice the kinetic energies, viz., about 0.8 and $0.4 \mathrm{eV}$, respectively. Thus, to pass from water to isooctane, Ps would have to overcome a threshold of some $0.4 \mathrm{eV}$, which possibly explains why, with $h$ $=0.1205 \mathrm{~nm}^{-1}$, the water spheres appear to be not completely permeable. However, a correct value of the threshold should take into account other effects, such as the charge-dipole interactions between the polar heads of the AOT molecules and Ps.

C. Variations in o-Ps Intensities in the AOT/Water/ Isooctane Mixtures. Qualitatively, the o-Ps intensity derived in three-component analysis of the LS spectra initially shows a rapid decrease when $C_{\mathrm{AOT}}$ increases at constant $w_{0}$ and then levels off to a value close to $I_{3}^{\mathrm{aq}}$. This behavior has been observed in previous work $^{8,29}$ and was attributed to some inhibiting effect of the micellar aggregates on Ps formation. However, due to the small value of $\tau_{3}$ as compared to $\tau_{4}$, the averaging implicit when imposing three components may result in a loss of calculated o-Ps intensity relative to the free $\mathrm{e}^{+}$ component, making further analysis difficult. In four-component analysis, both $I_{3}$ and $I_{4}$ decrease very rapidly with increasing $C_{\mathrm{AOT}}$ (see Table 1). It is noticeable that the total o-Ps intensity, $I_{\text {tot}}$, which, therefore, also decreases, reaches a value close to $I_{3}^{\text {aq }}$. It can be seen in particular that, for $C_{\mathrm{AOT}}>0.04 \mathrm{M}, I_{\mathrm{tot}}$ remains close to or slightly lower than $I_{3}^{\text {aq }}$, independent of $C_{\mathrm{AOT}}, w_{0}$, and $T$ as illustrated in Figure 4 . An explanation of the decrease in the Ps intensity in terms of inhibition by the micelles ${ }^{7,29,30}$ appears rather unlikely for several reasons: (i) Sodium methanesulfonate shows only a very low tendency to inhibit Ps formation. (ii) The concentration of the micellar aggregates is very low. For example, for $C_{\mathrm{AOT}}=0.1 \mathrm{M}$ and $w_{0}=20$ at $294 \mathrm{~K}$, eq A13 gives a micelle concentration of only $3 \times 10^{-4} \mathrm{M}$. Species present at such a low concentration, and occupying a correspondingly very small volume fraction, are unlikely to be reactive enough, in isooctane, to efficiently scavenge the positron or the electron in the spur within the very short time scale for Ps formation. (iii) In addition, the $\tau_{3}$ variations, referred to earlier, indicate efficient diffusion to the organic phase of those Ps initially formed in the aqueous cores, $\mathrm{Ps}^{\mathrm{aq}}$, resulting in the increase in $I_{4}$ at the cost of $I_{3}$. Therefore, it is suggested that direct Ps formation in isooctane is quite inefficient, at least for values of $w_{0}>10$.

The similarity between the values of $I_{\text {tot }}$ and $I_{3}^{\mathrm{aq}}$ strongly suggests that Ps formation occurs primarily in the aqueous phase. This implies that a large fraction of those positrons that would have formed Ps in pure isooctane is now trapped by the aqueous micellar droplets, where they form Ps. Due to the small volume fraction of the micelles, it is unlikely that the trapping of the positrons would occur in competition with Ps formation in the spurs created inside isooctane, firstly because, geometrically, the probability of overlap between the small spur region and the micelle is certainly too low, and secondly, on thermodynamic grounds the positron would not have sufficient energy to ionize the water if it were trapped at the end of its track, i.e., nearly thermalized. Therefore, it must be concluded that the positrons are trapped in water while they are slowing down in isooctane. The $\mathrm{e}^{+}$energy window within which the process occurs must be such that the positrons cannot escape from the aqueous cores, but still possess sufficient energy to ionize water and form Ps.
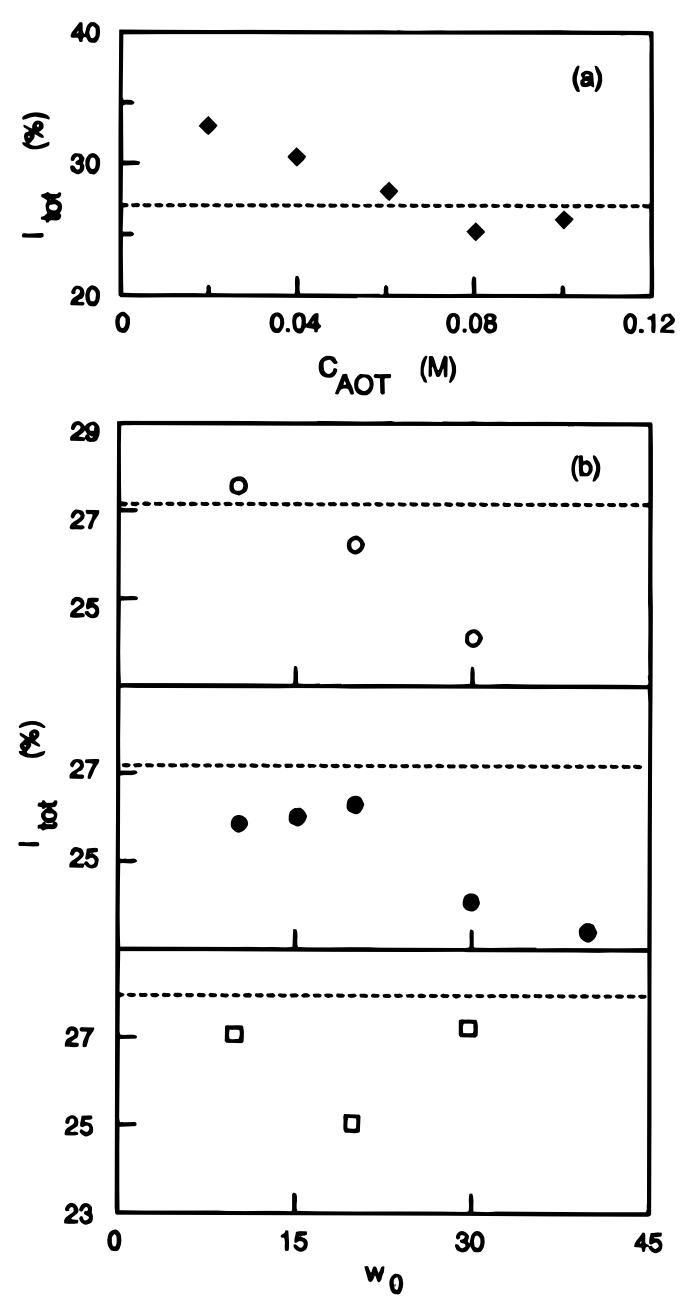

Figure 4. Variation in the total o-Ps intensity, $I_{\text {tot }}=I_{3}+I_{4}(\%)$, as a function of (a) AOT concentration in isooctane, $C_{\mathrm{AOT}}(\mathrm{M})$, at $294 \mathrm{~K}$; (b) water/AOT mole ratio, $w_{0}$, at various temperatures, $T(\mathrm{~K})=294$ (○), $298(\bullet)$, and $323(\square)$. The dashed lines correspond to the respective values of $I_{3}^{\text {aq }}$ for pure water.

On the time scale of $\mathrm{e}^{+}$slowing down (ca. picoseconds) little movement of solvent molecules occurs, and the microemulsion medium can be treated as an amorphous solid. For solids, the average distance, $x$, traveled by positrons of maximum energy, $E_{\max }(\mathrm{MeV})$, in a medium of density, $d$, is given by ${ }^{31}$

$$
x(\mathrm{~cm})=E_{\max }^{1.43} / 16 d
$$

Since the water/isooctane density ratio is $1 / 0.69$, the water droplets have an efficiency to stop the slowing down positrons that is some $30 \%$ higher than that of spherical isooctane regions of the same radius. For illustration, the average distance traveled by an energetic particle through a sphere of radius $r_{0}=3.6 \mathrm{~nm}$ is $x=3.6 \pi / 2=5.65 \mathrm{~nm}$. According to eq 11 , such a sphere would be able to efficiently stop positrons of maximum energies of about 300 and only $230 \mathrm{eV}$ in water and isooctane, respectively. The particular efficiency of the water aggregates compared to those of isooctane in slowing down and trapping the positrons may also partly arise from charge-charge interactions between $\mathrm{e}^{+}$and the polar headgroups of the AOT molecules and from the charge-dipole interactions with the water molecules, the latter eventually leading to localization and solvation of the positrons.

Quantitatively, Ps formation in water thus may originate both from those positrons that happen to form a spur in water, giving a Ps formation probability proportional to the water volume 


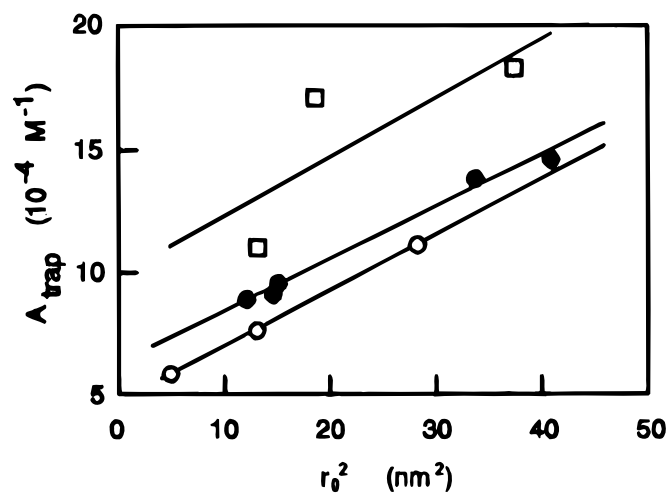

Figure 5. Variation in $A_{\text {trap }}$, from eq A14, with $r_{0}^{2}\left(\mathrm{~nm}^{2}\right)$ at various temperatures $T(\mathrm{~K})=294(\bigcirc), 298(\bullet)$, and $303(\square)$. For the solid lines, see text.

fraction and from the positrons trapped while slowing down in isooctane. The relevant equations are given in the Appendix for $I_{3}$ and $I_{4}$, with the trapping constant, $k_{\text {trap }}$, being an explicit function of the micelle concentration, $k_{\text {trap }}=A_{\text {trap }} C_{\text {mic }}$. As $I_{\text {tot }}$ reaches values that are slightly lower than $I_{3}^{\text {aq }}$ (Table 1 ), the possibility of some inhibition by the AOT molecules in the micelles is also included. Thus, each set of values for $I_{3}$ and $I_{4}$ as given in Table 1 can be fitted using two parameters: $A_{\text {trap }}$ and the inhibition constant, $k$ (see eq A10). The results are given in Table 1 for $k$ at $C_{\mathrm{AOT}}=0.1 \mathrm{M}$, while Figure 5 shows the variation of $A_{\text {trap }}$ with $r_{0}^{2}$ at 294 and $298 \mathrm{~K}$, temperatures at which the data are best determined, and also at $303 \mathrm{~K}$. It may be seen that $A_{\text {trap }}$ varies linearly with $r_{0}{ }^{2}$, with a slope that is independent of $T$. Fitting of the data gives slopes of 2260, 2113, and $2376 \mathrm{~nm}^{-2} \mathrm{M}^{-1}$ at 294, 298, and $303 \mathrm{~K}$, respectively. Note that the non-zero intercept of the lines is probably due, in part, to the fact that, as shown by other techniques, ${ }^{14}$ "pure" AOT, with $w_{0}=0$, does contain some associated water molecules. The behavior of $k_{\text {trap }}$ with $C_{\text {mic }}, r_{0}$, and $T$ lends strong support to the hypothesis of capture by the water aggregates of energetic positrons, with an efficiency that should depend on the cross section of the aggregates (i.e., proportional to $r_{0}^{2}$ ) and that should show little sensitivity to $T$. At $T$ above $303 \mathrm{~K}$, the data on $\tau_{3}$, which are experimentally correlated with $I_{3}$ and also serve implicitly, through the calculation of $r_{0}$, to evaluate both $I_{3}$ and $I_{4}$, are somewhat too disperse to allow a precise determination of $k_{\text {trap. }}$

From Table 1, it can be seen that the values of $k$ are poorly defined, as they are strongly dependent on the value of $I_{3}$. As a consequence, only some general features will be discussed in the following.

Alkyl sulfates and sulfonates have very poor electron scavenging abilities. ${ }^{32,33}$ Considering the correlations ${ }^{3}$ previously found between the Ps inhibition constants of solutes and their reaction rate constants with the solvated electron, $k\left(\mathrm{e}_{\mathrm{aq}}^{-}\right)$, the values of $k$ in Table 1 would effectively correspond to $k$ $\left(\mathrm{e}_{\mathrm{aq}}^{-}\right)<10^{8} \mathrm{M}^{-1} \mathrm{~s}^{-1} \cdot 3,34$ Qualitatively, the data appear to indicate little influence of $T$ on $k$, in agreement with inhibition through scavenging of nonsolvated electrons. In contrast, if $\mathrm{e}^{+}$ scavenging were involved, then the corresponding $\mathrm{e}^{+}$bound state with AOT should have a shorter lifetime than free $\mathrm{e}^{+}$. This is in agreement with the decrease in $\tau_{2}$ observed in micellar systems as compared to either pure water or isooctane (see eq 6 ). However, a significant effect of the temperature on $k$ would be expected, ${ }^{35}$ and this is not observed.

In general, the data show a tendency for $k$ to increase with $w_{0}$. This requires further investigation. Theoretically, this possibility is difficult to quantify, as inhibition occurs in a nonhomogeneous system, with the active polar heads distributed at the surface of the water spheres and the $\mathrm{e}^{-}$and $\mathrm{e}^{+}$in the water spur.

\section{Conclusion}

The results presented show that new information can be derived by LS on both the micellar systems and the probes. Regarding the latter, two important characteristics are evidenced: (i) Efficient trapping of the positrons by the water aggregates while they are slowing down in the organic solvent leads to an apparent decrease in the total Ps yield, because some proportion of positrons form Ps in water with a probability that is substantially lower than in isooctane $\left(I_{3}^{\text {aq }}<I_{3}^{\text {is }}\right)$; this increases with the size of the micelles. (ii) The subsequent diffusion to the organic solvent of the Ps atoms formed in water results in a decrease in the Ps lifetime in the micelles. Solvation of the appropriate, rigorous equations for this out-diffusion process leads to quantitative information on the radius of the aqueous cores of the micelles.

The $\mathrm{e}^{+}$trapping and Ps out-diffusion processes should be confirmed in further experiments. To this end, various ways are open that should enrich our knowledge of both the probes and the micelles. For example, examination of Ps inhibition by using electron scavenging solutes specifically soluble in the organic phase should give precise information on the relative amounts of Ps either that are directly formed in the organic phase or that have been created in the water aggregates before diffusing out to the organic phase. Similarly, the Ps diffusion, through which the size of the water cores can be inferred, could be studied in other micellar systems, whose structures are known sufficiently from other techniques to allow quantitative comparison with the information obtained in LS. In this respect, systems presenting larger water core radii than those in the present study, leading to longer Ps lifetimes and, therefore, to more precise LS parameters, appear promising.

Although the inhibiting power of the AOT molecules is rather weak, this should also be examined further. Notwithstanding the difficulties inherent to a system containing many positron states, experiments using Doppler broadening or angular correlation techniques might shed some light on the nature of the inhibition reactions, either by $\mathrm{e}^{+}$or by $\mathrm{e}^{-}$scavenging, through the search for any $\mathrm{e}^{+}$bound state formation.

Acknowledgment. Part of this work was carried out within the framework of the Programa de Cooperação Cientifica $e$ Técnica Luso-Francês (JNICT/French Ambassy in Lisbon).

\section{Appendix. Calculation of Lifetime Spectroscopy Parameters for Four-Component Analysis in AOT/Water/ Isooctane Microemulsions}

The Second Longest Lifetime, $\boldsymbol{\tau}_{3}$. The general time equation for Ps ${ }^{\text {aq }}$ diffusing out of a sphere of radius $r_{0}$, with diffusion coefficient $D_{\mathrm{Ps}}$, and decaying with rate constant $\lambda_{3}^{\mathrm{aq}}$ is given by $^{21}$

$$
\mathrm{Ps}^{\mathrm{aq}}=\int_{0}^{r_{0}} \sum_{n=0}^{\infty} A_{n} \exp \left(-\Lambda_{n} t\right) 4 \pi r \sin \left(\lambda_{n} r\right) \mathrm{d} r
$$

with $A_{n}=\left(2 / r_{0}\right) B C_{0} \int_{0}^{r_{0}} r \sin \left(\lambda_{n} r\right) \mathrm{d} r, B=\left[\lambda_{n}{ }^{2} r_{0}^{2}+\left(r_{0} h-1\right)^{2}\right] /$ $\left[\lambda_{n}{ }^{2} r_{0}{ }^{2}+r_{0} h\left(r_{0} h-1\right)\right]$, and $\Lambda_{n}=\lambda_{n}{ }^{2} D_{\mathrm{Ps}}+\lambda_{3}^{\mathrm{aq}} . C_{0}$ is the initial spatial distribution of the Ps atoms; normalization to one Ps particle randomly created in the sphere gives $C_{0}=3 /\left(4 \pi r_{0}{ }^{3}\right)$. The $\lambda_{n}$ 's are the successive positive roots of 


$$
\operatorname{tg}\left(\lambda_{n} r_{0}\right)=\lambda_{n} r_{0} /\left(1-r_{0} h\right)
$$

Parameter $h$ is a transmission factor, such that the sphere is impermeable for $h=0$ and totally permeable for $h \rightarrow \infty$. Rearrangement of eq A1 leads to

$$
\operatorname{Ps}^{\mathrm{aq}}=6\left(r_{0} h\right)^{2} \sum_{n=1}^{\infty} F(x) \exp \left(-\Lambda_{n} t\right)
$$

where $x=\lambda_{n} r_{0}$, solution of eq A2, $\Lambda_{n}=\left(x^{2} D_{\mathrm{Ps}} / r_{0}^{2}+\lambda_{3}^{\mathrm{aq}}\right)$, and $1 / F(x)=x^{2}\left[x^{2}+r_{0} h\left(r_{0} h-1\right)\right]$.

Knowledge of $\mathrm{Ps}^{\mathrm{aq}}$ allows one to derive the theoretical expression describing the LS spectra given by $\mathrm{d}(2 \gamma) / \mathrm{d} t$ on the basis of eq 7. To this end, the equation for $\mathrm{Ps}^{\text {is }}$ must be established. In the absence of decay in isooctane, that fraction of Ps atoms, Ps ${ }^{\text {out }}$, that at time $t$ neither would be present in the micelles $\left(\mathrm{Ps}^{\mathrm{aq}}\right)$ nor would have decayed in the micelles is given by

$$
\begin{aligned}
& \mathrm{Ps}^{\text {out }}=1-\mathrm{Ps}^{\mathrm{aq}}-\int_{0}^{t} \lambda_{3}^{\mathrm{aq}} \mathrm{Ps}^{\mathrm{aq}} \mathrm{d} t= \\
& 1-\mathrm{Ps}^{\mathrm{aq}}-6\left(r_{0} h\right)^{2} \lambda_{3}^{\mathrm{aq}} \sum_{n=1}^{\infty} F(x) G(x)\left[1-\exp \left(-\Lambda_{n} t\right)\right]
\end{aligned}
$$

with $G(x)=1 / \Lambda_{n}$ and $\mathrm{Ps}^{\mathrm{aq}}, F(x)$, and $\Lambda_{n}$ as defined in eq A3.

Since o-Ps is to decay in isooctane with a rate constant $\lambda_{3}^{\text {is }}$, $\mathrm{Ps}^{\text {is }}$ is given by the convolution of $\mathrm{dPs}^{\text {out}} / \mathrm{d} t$ with the decay function, $\exp \left(-\lambda_{3}^{\text {is }} t\right)$, leading to

$$
\mathrm{Ps}^{\text {is }}=6\left(r_{0} h\right)^{2} \sum_{n=1}^{\infty} F(x) H\left(\Lambda_{n}\right)\left(\mathrm{e}^{-\Lambda_{n} t}-\mathrm{e}^{-\lambda_{3}^{\text {is } t}}\right)
$$

with $H\left(\Lambda^{n}\right)=\left(\Lambda_{n}-\lambda_{3}^{\mathrm{aq}}\right) /\left(\lambda_{3}^{\text {is }}-\Lambda_{n}\right)$.

Finally, the LS spectrum may be quantified by

$$
\mathrm{d}(2 \gamma) / \mathrm{d} t=\lambda_{3}^{\mathrm{aq}} \mathrm{Ps}^{\mathrm{aq}}+\lambda_{3}^{\mathrm{is}} \mathrm{Ps}^{\mathrm{is}}
$$

Note that, in this equation, only those Ps atoms (normalized to 1 at $t=0$ ) that have been formed in water and eventually diffused out to isooctane are considered; those Ps atoms initially formed in isooctane will merely decay with rate constant $\lambda_{3}^{\text {is }}$ and, thus, do not interfere in the expression of $\tau_{3}$. From eqs A3 and A5, the LS spectrum includes two components, with decay rate constants $\Lambda_{n}$ and $\lambda_{3}^{\text {is }}$. The latter strictly corresponds to o-Ps decay in pure isooctane, so that the second longest lived component of the spectrum, $\mathrm{d}(2 \gamma) / \mathrm{d} t\left(\Lambda_{n}\right)$, is obtained by summing the exponential terms associated to decay rate constant $\Lambda_{n}$ :

$$
\mathrm{d}(2 \gamma) / \mathrm{d} t\left(\Lambda_{n}\right)=6\left(r_{0} h\right)^{2} \sum_{n=1}^{\infty} F(x)\left[\lambda_{3}^{\mathrm{aq}}+H\left(\Lambda_{n}\right) \lambda_{3}^{\mathrm{is}}\right] \exp \left(-\Lambda_{n} t\right)
$$

The experimental decay rate constant, $\lambda_{3}$, therefore represents the average decay rate constant of the $n$ terms in eq A7. Each of these terms has an intensity, $I_{n}$, given by

$$
\begin{array}{r}
I_{n}=\int_{0}^{\infty} 6\left(r_{0} h\right)^{2} F(x)\left[\lambda_{3}^{\mathrm{aq}}+\lambda_{3}^{\mathrm{is}} H\left(\Lambda_{n}\right)\right] \exp \left(-\Lambda_{n} t\right) \mathrm{d} t= \\
6\left(r_{0} h\right)^{2} F(x)\left(\lambda_{3}^{\text {is }}-\lambda_{3}^{\mathrm{aq}}\right) /\left(\lambda_{3}^{\text {is }}-\Lambda_{n}\right)
\end{array}
$$

so that

$$
\begin{aligned}
1 / \tau_{3}=\lambda_{3}= & \sum_{n=1}^{\infty} I_{n} \Lambda_{n} / \sum_{n=1}^{\infty} I_{n}= \\
& {\left[\sum_{n=1}^{\infty} F(x) \Lambda_{n} /\left(\lambda_{3}^{\text {is }}-\Lambda_{n}\right)\right] /\left[\sum_{n=1}^{\infty} F(x) /\left(\lambda_{3}^{\text {is }}-\Lambda_{n}\right)\right] }
\end{aligned}
$$

Intensities $\boldsymbol{I}_{\mathbf{3}}$ and $\boldsymbol{I}_{\mathbf{4}}$. The intensities are governed by the following processes: (i) casual existence of a positron spur in either phase, leading to Ps formation in proportion to the intensities in the neat liquids, $I_{3}^{\mathrm{aq}}$ and $I_{3}^{\mathrm{is}}$, for water and isooctane, respectively, and to the corresponding volume fractions, $V_{\mathrm{aq}}$ and $\left(1-V_{\mathrm{aq}}\right)$, respectively; (ii) capture of the positrons by the water aggregates, with a trapping constant, $k_{\text {trap }}$, that is an explicit function of the aggregate concentration, $C_{\text {mic }}$; (iii) inhibition of Ps formation in the micelles, with inhibition constant $k$; and (iv) diffusion of Ps from the aqueous to the organic phase.

This leads to the following equation for $I_{3}$ :

$$
I_{3}=\left[I_{3}^{\mathrm{aq}} V_{\text {aq }}+I_{3}^{\mathrm{aq}}\left(1-V_{\mathrm{aq}}\right) P_{\text {trap }}\right] F_{\text {inhib }} P_{\text {in }}
$$

which can be rearranged as

$$
I_{3}=I_{3}^{\mathrm{aq}}\left[V_{\text {aq }}+\left(1-V_{\mathrm{aq}}\right) P_{\text {trap }}\right] F_{\text {inhib }} P_{\text {in }}=I_{3}^{0} P_{\text {in }}
$$

where $I_{3}^{0}$ denotes that fraction of o-Ps effectively created in water before diffusion occurs. The fractional volume occupied by water, $V_{\mathrm{aq}}$, and the inhibition function, ${ }^{3} F_{\text {inhib }}$, are given by

$$
\begin{gathered}
V_{\mathrm{aq}}=18 w_{0} C_{\mathrm{AOT}} / 1000 \\
F_{\text {inhib }}=1 /\left(1+k C_{\mathrm{AOT}}^{\mathrm{aq}}\right)
\end{gathered}
$$

where the AOT concentration in water is given by $C_{\mathrm{AOT}}^{\mathrm{aq}}=$ $1000 /\left(18 w_{0}\right)$.

The trapping probability, $P_{\text {trap }}$, is given by $P_{\text {trap }}=k_{\text {trap }} /(1+$ $\left.k_{\text {trap}}\right)$, where $k_{\text {trap }}$ is an explicit function of the micelle concentration:

$$
C_{\text {mic }}=C_{\mathrm{AOT}} w_{0} 18 /\left(4 \pi r_{0}^{3} d N_{\mathrm{Av}} / 3\right)
$$

with $d$ being the water density at specified $T$ and $N_{\mathrm{Av}}$ the Avogadro number, so that

$$
k_{\text {trap }}=A_{\text {trap }} C_{\mathrm{AOT}} w_{0} /\left(d r_{0}^{3}\right)
$$

Finally, $P_{\text {in }}$ in eq A10 is the contribution of those o-Ps atoms formed in water to the component with lifetime $\tau_{3}$ and is given by eq $\mathrm{A} 8$ as

$$
P_{\text {in }}=\sum_{n=1}^{\infty} I_{n}
$$

By knowing $I_{3}, I_{4}$ is obtained by subtracting $I_{3}$ from the total o-Ps yield:

$$
\begin{aligned}
& I_{4}=I_{3}^{\text {is }}\left(1-V_{\text {aq }}\right)\left(1-P_{\text {trap }}\right)+I_{3}^{0}-I_{3}= \\
& I_{3}^{\text {is }}\left(1-V_{\text {aq }}\right)\left(1-P_{\text {trap }}\right)+I_{3}^{0}\left(1-P_{\text {in }}\right)
\end{aligned}
$$

\section{References and Notes}

(1) Positron and Positronium Chemistry; Schrader, D. M., Jean, Y. C., Eds.; Elsevier: Amsterdam, 1988.

(2) Mogensen, O. E. J. Chem. Phys. 1974, 60, 998.

(3) Abbé, J. Ch.; Duplâtre, G.; Maddock, A. G.; Talamoni, J.; Haessler, A. J. Inorg. Nucl. Chem. 1981, 43, 2603. 
(4) Duplâtre, G. In Positron and Positronium Chemistry; Jean, Y. C., Ed.; World Scientific: Singapore, 1990; p 329.

(5) Positron and Positronium Chemistry; Jean, Y. C., Ed.; World Scientific: Milwaukee, WI, 1990.

(6) Positron and Positronium Chemistry (Billard, I., Ed.). J. Phys. IV 1993, 3 .

(7) Alfassi, Z. B.; Ache, H. J. J. Phys. Chem. 1984, 88, 4347.

(8) Djermouni, B.; Ache H. J. J. Phys. Chem. 1979, 83, 2476.

(9) Zulauf, M.; Eicke, H. F. J. Phys. Chem. 1979, 83, 480.

(10) Goto, A.; Harada, S.; Fujita, T.; Miwa, Y.; Yoshioka, H.; Kishimoto, H. Langmuir 1993, 86-89, 86.

(11) Dijk, M. A.; Joosten, J. G. H.; Levine, Y. K.; Bedeaux, D. J. Phys. Chem. 1989, 93, 2506.

(12) Belletete, M.; Lachapelle, M.; Durocher, G. J. Phys. Chem. 1990, $94,5337$.

(13) Wong, M.; Thomas, J. K.; Gratzel, M. J. Am. Chem. Soc. 1976, 98, 2391.

(14) Eastoe, J.; Fragneto, G.; Robinson, B. H.; Towey, T. F.; Heenan,

R. K.; Leng, F. J. J. Chem. Soc., Faraday Trans. 1 1992, 88, 461.

(15) Haessler, A.; Abbé, J. Ch.; Duplâtre, G. Nucl. Instrum. Methods 1980, $174,317$.

(16) Didierjean, F.; Billard, I.; Magalhaes, W. F.; Duplâtre, G. Chem. Phys. 1993, 174, 331 .

(17) Mogensen, O. E.; Jacobsen, F. M. Chem. Phys. 1982, 73, 223.

(18) Talamoni, J.; Abbé, J. Ch.; Duplâtre, G.; Haessler, A. Radiat. Phys. Chem. 1982, 20, 275.

(19) Duplâtre, G.; Haessler, A.; Abbé, J. Ch. J. Phys. Chem. 1985, 89, 1756.

(20) Aprano, A. D.; D’Arriago, G.; Paparelli, A.; Goppedi, M.; Livesi, V. T. J. Phys. Chem. 1993, 97, 3614.

(21) Budak, B. W.; Tikhonov, A. N.; Samarskii, A. A. The Equations of Mathematical Physics; IZd, Nauka: Moscow, 1972; p 517.
(22) Magalhaes, W. F.; Abbé, J. Ch.; Duplâtre, G. Struct. Chem. 1991, 2, 399.

(23) Robinson, B. H.; Toprakcioglu, C.; Dore, J. C.; Chieux, P. J. Chem. Soc., Faraday Trans. 1 1984, 80, 13.

(24) Izquierdo, C.; Moya, M. L.; Usero, J. L.; Casado, J. Monatsh. Chem. 1992, 123, 383.

(25) Luisi, P. L.; Giomini, M.; Pileni, M.; Robinson, B. H. Biochim. Biophys. Acta 1988, 947, 209.

(26) Day, R. A.; Robinson, B. H. J. Chem. Soc., Faraday Trans. 11979 $75,132$.

(27) Kotlarchyk, M.; Chen, S.-H.; Huang, J. S. J. Phys. Chem. 1982, $86,3273$.

(28) Billard, I.; Abbé, J. Ch.; Duplâtre, G. J. Phys. Chem. 1991, 95, 2501.

(29) Ferreira Marques, M. F.; Lopes Gil, C.; de Lima, A. P.; Burrows, H. D.; da Graça Miguel, M. J. Phys. IV 1993, 3, 317.

(30) Boussaha, A.; Djermouni, B.; Fucugauchi, L. A.; Ache, H. J. J. Am. Chem. Soc. 1980, 102, 4654.

(31) Nieminen, R. M.; Manninen, M. J. In Positrons in Solids; Hautojärvi, P., Ed.; Springer-Verlag: 1979; p 145.

(32) Anbar, M.; Bambenek, M.; Ross, A. B. National Bureau of Standards, NSRDS-NBS 43, Part 1, 1973.

(33) Ross, A. B. National Bureau of Standards, NSRDS-NBS 43, Supplement, 1975.

(34) Duplâtre, G.; Jonah, C. D. Radiat. Phys. Chem. 1985, 24, 557.

(35) Talamoni, J.; Duplâtre, G.; Abbé, J. Ch.; Haessler, A. Chem. Phys. 1984, 83,471 .

JP952838S 